\title{
Chemotherapy Regimen in Nonagenarian Cancer Patients: A Bi-Institutional Experience
}

\author{
Romain Rivoirard $^{\mathrm{a}}$ Cyrus Chargari ${ }^{\mathrm{b}}$ Sharif Kullab ${ }^{c}$ Jane-Chloé Trone ${ }^{\mathrm{d}}$ \\ Julien Langrand-Escure ${ }^{d}$ Guillaume Moriceau ${ }^{a}$ Jean-Baptiste Guy ${ }^{d}$ Pierre Annede ${ }^{a}$ \\ Benoîte Méry $^{a}$ Coralie Moncharmont $^{d} \quad$ Alexander Tuan Falk $^{\mathrm{e}}$ Lionel Vedrine $^{\mathrm{b}}$ \\ Yacine Merrouche $^{c}$ Pierre Fournel $^{c}$ Nicolas Magnéd \\ ${ }^{a}$ Medical Oncology, Jean Perrin Comprehensive Cancer Center, Clermont-Ferrand, ${ }^{\mathrm{b}}$ Medical and Radiation \\ Oncology, Hôpital d'Instruction des Armées du Val-de-Grâce, Paris, ' Medical Oncology and d Radiation Oncology, \\ Institut de Cancérologie Lucien Neuwirth, Saint-Priest-en-Jarez, and ${ }^{\circledR}$ Radiation Oncology, Antoine Lacassagne \\ Comprehensive Cancer Center, Nice, France
}

\section{Key Words \\ Chemotherapy · Nonagenarians · Radiotherapy}

\begin{abstract}
Background: The elderly population in Western countries is growing and constitutes a public health issue. Concomitantly, age-related diseases such as cancer increase. There are few data on the efficacy, tolerability and toxicity of specific anticancer therapy in the very elderly patients; therefore, their management is not standardized. Methods: In this biinstitutional study, we reviewed medical records of patients who received or continued specific anticancer therapy beyond the age of 90 years. Geriatric assessment was not reported for our patients. Twelve patients were enrolled. Their general health condition was good, and half of them were living in elderly institutions. Ten patients had a solid tumor and 2 were treated for hematological malignancies. Most were diagnosed with a locally advanced or metastatic disease, and the goal of treatment was curative for only 1 patient. Six patients received chemotherapy as first-line treatment, 4 patients received targeted therapy and 2 received concomitant chemoradiation. Four patients received a sec-
\end{abstract}

ond-line treatment. Results: Despite a significant reduction in treatment posology in half of the patients, 8 acute grade $3 / 4$ toxicities were reported and 2 patients died of treatment-related septic shock. Median duration of first-line treatment was 3.2 months, and progression-free survival ranged from 18 to 311 days. Overall survival ranged from 18 days to 11 years. Conclusion: Aging is a heterogeneous process, and management of elderly patients is a multidisciplinary approach. Geriatric assessment helps to identify older patients with a higher risk of morbidity/mortality and allows to assess the risks and benefits of specific anticancer therapy. The choice of treatment should be based primarily on the expected symptomatic benefit, and treatment should not compromise the quality of life.

(c) 2015 S. Karger AG, Basel

\section{Introduction}

Despite the large proportion of elderly patients with cancers, several studies have shown that older patients are not treated to the same extent as young patients. Moreover, they are often excluded or underrepresented in clin-

\section{KARGER}

E-Mail karger@karger.com

www.karger.com/che (c) 2015 S. Karger AG, Basel

0009-3157/15/0612-0065\$39.50/0
Prof. Nicolas Magné, MD, $\mathrm{PhD}$

Radiation Oncology, Institut de Cancérologie Lucien Neuwirth 108 bis, avenue Albert Raimond, BP 60008

FR-42271 Saint-Priest-en-Jarez (France)

E-Mail nicolas.magne@icloire.fr 
ical trials, and among those who do enroll, detailed data regarding comorbid conditions and psychological factors are not routinely captured [1-6]. Beside their underrepresentation, studies which include older patients are likely to have selection bias 'only fit elderly patients were included' $[4,7]$.

Most clinical studies consider a chronological age of 75 years for treatment inclusion [8]. However, chronological age alone is not a sufficient factor to withhold curative or palliative treatment from an elderly patient. Other factors like more frequent morbidities, poor functional reserves, social support, precarious physical conditions and mental status should be considered $[3,9]$.

Elderly patients are more prone to experience toxicities to specific anticancer treatment. This risk of toxicities increases with age, comorbidities and, to a certain extent, the treatment of the patient's other comorbidities. The treating oncologist should evaluate elderly patients and then select those who would benefit from a standard oncology treatment.

Tools such as comprehensive geriatric assessment in hospital settings can identify many health and functional state issues that might not otherwise be known by the treating oncologist. In addition, several domains of geriatric assessment are associated with oncological outcomes, such as toxicity of treatment and mortality [10]. Using this evaluation, physicians could identify 'fit' patients who can receive the same treatment as that offered to young patients, also identify 'vulnerable' patients who need a tailored treatment and finally 'frail' patients who will probably not tolerate a radical therapy proper to their diseases [3].

Inclusion of frail elderly patients in treatment trials is recommended. New treatment approaches for frail elderly cancer patients need to be further investigated [11].

The objective of this study is to report the epidemiological and clinical data of our cancer patients older than 90 years who were treated with chemotherapy and followed up in two cancer centers, and to discuss the ethical and deontological aspects of chemotherapy in this setting of patients.

\section{Patients and Methods}

This is a retrospective cohort study conducted in two medical oncology and hematology departments of public health institutions that have a university hospital mission: the Lucien Neuwirth Cancerology Institute and the Teaching Military Hospital of Valde-Grâce. Of about 2,800 new patients yearly diagnosed and treat- ed for their cancers, 12 patients underwent or followed a specific anticancer treatment beyond the age of 90 years between January 2005 and December 2013.

Inclusion of the patients was possible through the computer databases of the Intravenous Chemotherapy Prescription Software (BPC: Bonnes Pratiques en Chimiothérapie). Clinical notes were taken during each patient's visit. Analysis of the patients' medical records was based on initial clinical data and medical notes retrieved either from hospital databases or archived medical records. However, for any further information, family physicians, referring doctors or physicians of long-stay settings were contacted. Marital status departments of patients' hometown municipalities were contacted as well in order to get some information about our patients if that was not noted in the patient files.

The patients' data included age, gender and performance status. However, no geriatric evaluation was assessed in our patients. The decision for anticancer treatments is being taken during multidisciplinary cancer meetings.

Patients' cancers were specified by their type, stage of the disease (localized, locally advanced or metastatic) and metastatic sites.

The global objective of treatment and different types of specific anticancer therapies were detailed. Chemotherapy/target therapy cycles and the lines of treatment were counted as well.

All death cases related to treatment were reported and all toxicities related to anticancer therapies were documented as well and were specified in grades from 1 to 4 .

\section{Results}

\section{Patients and Their Cancers}

Twelve patients started or continued their anticancer treatment beyond the age of 90 . There were 8 females and 4 males. Their age ranged from 85 to 93 years, with a mean age of around 90.5 years. The whole patients enrolled in our study were found to have maintained general life conditions. However, half of them were living in elderly institutions at the time of the diagnosis of their cancers. Among the 12 patients, 3 were fully active, able to carry on all predisease performance without restriction [ECOG performance status (PS) $=0$ ]. Seven patients were mildly restricted in physical strenuous activities but still ambulatory and able to carry out work of light nature ( $P S=1)$ and the remaining 2 patients had a PS of 2.

Ten patients were found to have a solid tumor ( 4 digestive tumors, 3 gynecological tumors and 1 case each of cutaneous squamous cell carcinoma, breast adenocarcinoma and clear cell renal carcinoma). The other 2 patients had a malignant hemopathy (1 case of chronic lymphoid leukemia and 1 case of non-Hodgkin lymphoma).
Rivoirard et al. 
At the moment of the diagnosis, only 4 patients had localized or low-grade disease. Most of the patients had either locally advanced (2 patients) or metastatic disease (6 patients). Metastatic sites were represented essentially by hepatic lesions (4 patients), lung metastases (3 patients), bone invasion (2 patients) and lymph nodes (2 patients).

The characteristics of the patients and their cancers are listed in table 1.

\section{Treatment}

The objective of the specific anticancer therapy in our nonagenarian patients was considered as palliative in most of the cases. It was curative in only 1 case of stage T2a cervical basaloid carcinoma, which was treated with concurrent chemoradiation.

In spite of their age, different types of anticancer therapy were offered. Five patients underwent surgical intervention and 1 patient had a palliative biliary endo-prosthesis. Chemotherapy alone was given to 6 patients as first-line treatment: therapy was based on capecitabine in 3 cases, a combination of carboplatin (AUC 5) and cyclophosphamide in 1 patient, Gemzar monotherapy in 1 patient and carboplatin (AUC 5) monotherapy in 1 patient. Furthermore, 2 patients received weekly carboplatinbased chemotherapy concurrently with radiation therapy in order to treat a squamous cell carcinoma patient and a cervical basaloid carcinoma patient. Three patients received radiation therapy for their symptomatic bone metastasis. Regarding target therapy, the first-line treatments consisted of rituximab for a non-Hodgkin lymphoma patient and a chronic lymphoid leukemia patient, temsirolimus for a clear cell renal carcinoma patient and trastuzumab for a case of breast cancer. Panitumumab was given as second-line treatment for 2 colorectal cancer patients.

However, it was necessary to reduce chemotherapy doses in half of the patients, either as precaution considering their age ( 5 patients) or because of a mild chronic renal failure (1 patient). These patients received $50 \%$ of the planned dose since the first cycle of their chemotherapy.

The number of treatment courses was variable between patients: 2 patients received less than 3 cycles, 4 patients received 3-6 cycles and 3 patients received 7-10 cycles. However, some target therapies were followed for at least 10 continuous courses in 3 other patients.

Half of our patients received only one line of treatment. Nevertheless, offering second-line treatment was estimated to be necessary in 4 patients. Two colorectal
Table 1. Characteristics of the patients and their cancers

\begin{tabular}{ll}
\hline Criteria & Patients, $\mathrm{n}$ \\
\hline Sex & \\
Women & 8 \\
Men & 4 \\
PS & 3 \\
0 & 7 \\
1 & 2 \\
2 & 0 \\
3 & 0 \\
4 & \\
Type of cancer & 4 \\
Digestive & 3 \\
Gynecological & 2 \\
Hematological & 1 \\
Breast & 1 \\
Dermatological & 1 \\
Renal & \\
Diagnostic stage & 4 \\
Localized & 2 \\
Locally advanced & 6 \\
Metastatic & \\
Metastatic sites & 4 \\
Liver & 3 \\
Lung & \\
Bone & \\
Lymph nodes & \\
\hline
\end{tabular}

carcinoma patients received capecitabine as first-line chemotherapy and then irinotecan combined with 5-fluorouracil and panitumumab as second-line therapy. One patient was treated for his pancreatic adenocarcinoma by oxaliplatin combined with 5 -fluorouracil as second-line treatment after first-line treatment with Gemzar. The 4th patient received oxaliplatin combined with 5 -fluorouracil after capecitabine therapy for his rectal carcinoma. Only 2 patients could have a third line of treatment. Table 2 shows treatment criteria and the different lines of treatment.

\section{Treatment Toxicity}

Table 3 describes the different treatment-emergent adverse events. The most frequent adverse events were of grade 1 or 2 (11 patients). The most common toxicity was diarrhea (4 patients) and nausea and vomiting ( 3 patients). The other treatment-related toxicities were less frequent: asthenia (2 patients), Gemzar-induced thrombocytopenia (1 patient) and atrial fibrillation (1 patient). 
Table 2. Treatment characteristics

\begin{tabular}{lc}
\hline Criteria & Patients, $\mathrm{n}$ \\
\hline Objective of the treatment & 1 \\
$\quad$ Curative & 11 \\
Palliative & 5 \\
Anticancer treatment & 6 \\
$\quad$ Surgery & 4 \\
First-line chemotherapy & 2 \\
First-line target therapy & 3 \\
Concurrent chemoradiation & 1 \\
Radiotherapy & 2 \\
Biliary prosthesis & 4 \\
First-line chemotherapy/target therapy cycles \\
$\quad$ < cycles & 3 \\
$3-6$ cycles & 3 \\
$7-10$ cycles & 6 \\
$>10$ cycles & \\
Chemotherapy dose reduction & 6 \\
Lines of treatment & 4 \\
1 line & 2 \\
2 lines & 0 \\
3 lines & \\
&
\end{tabular}

Table 3. Toxicity of treatment

\begin{tabular}{lc}
\hline Criteria & Patients, $\mathrm{n}$ \\
\hline Grade 1/2 toxicity & 11 \\
Diarrhea & 4 \\
Nausea/vomiting & 3 \\
Asthenia & 2 \\
Cardiac (atrial fibrillation) & 1 \\
Hematological & 1 \\
Grade 3/4 toxicity & 8 \\
Cardiac & 2 \\
Hematological & 2 \\
Sepsis & 1 \\
Arthromyalgia & 1 \\
Cutaneous & 1 \\
Asthenia & 1 \\
Septic shock and death & 2 \\
\hline
\end{tabular}

During treatment, grade 3 and 4 adverse events were reported in almost 8 patients and consisted essentially of hematological toxicities (1 case of rituximab-induced thrombocytopenia and 1 case of Gemzar-induced neutropenia) and cardiac toxicity (1 case of right heart impairment on rituximab and in 1 case of asymptomatic decrease in left ventricular ejection fraction $>18 \%$ after trastuzumab treatment). The other severe toxicities were asthenia following temsirolimus treatment, panitumumab-induced cutaneous toxicity, severe sepsis after carboplatin-based chemotherapy and arthromyalgia attributed to capecitabine.

Two patients died secondary to septic shock less than 1 month after initiation of the specific anticancer therapy, and their death was attributed to this treatment. One of them died after his first cycle of carboplatin combined with cyclophosphamide and the other died after receiving his 9th cycle of temsirolimus treatment.

\section{Survival}

The duration of first-line treatment extended from 0.5 to 9 months, with a median of 3.2 months. Progressionfree survival ranged from 18 days to 311 days in the firstline treatment and from 66 to 300 days in the second-line treatment. Overall survival varied between 18 days and more than 11 years.

\section{Discussion}

The frequency and the severity of toxicity secondary to anticancer therapy, and namely to chemotherapy, are more common in elderly than in younger patients, and can complicate their treatment [12].

The uncertain relation between age, comorbidities and toxicity is a major impediment to the inclusion of older patients in trials of drug safety and efficacy $[13,14]$. Certain common age-related physiological changes increase the likelihood of toxicity with chemotherapy: decreased ability to repair cell damages, decreased stem cell reserve and greater anemia, reduced functional reserve of organ systems with progressive loss of body protein and accumulation of body fat, decreased liver and nephron mass, and reduced gastric mobility and secretion, and gastrointestinal absorption surface [14]. The global decline in organ functions associated with age-related comorbidities, and possibly the drugs used to treat these comorbidities, increase the risk of toxicity of some chemotherapy agents on the body.

All these conditions obligate the oncologists to reduce the dose of chemotherapy in order to have less adverse events, but without being sure of the treatment efficacy at this reduced dose.

In our short cohort study, the reduction in chemotherapy posology started since the first cycle of treatment and reached almost $50 \%$ of the planned dose in half of the treated patients due to their age and anticipated treat-
68

Chemotherapy 2015-16;61:65-71 DOI: $10.1159 / 000441018$
Rivoirard et al. 
ment adverse events. Despite this adaptation of chemotherapy posology, almost two thirds of the patients presented serious adverse events of grade 3 or 4 . Furthermore, 2 patients died also secondary to their systemic treatment.

The development and use of elderly-specific regimens may produce better outcomes than those using an empirically reduced dose of a standard regimen [15]. However, few agents have been tested in phase II or III trials in older patients. The combination treatment not only did not increase survival over that achieved with each drug alone, it was also more toxic [16].

The small number of our patients could be explained partially by the recruitment procedures of screening campaigns that target usually a population of less than 75 years of age. Similarly, the very elderly patients are less often referred to medical oncologists than to family physicians and general geriatricians. This has been verified and confirmed in the different tumor boards through the patients' systemic medical records since January 2005. In fact, the patients in our study were referred directly by their family physicians to medical oncologists. This reflects a degree of bias regarding patient selection criteria. Another limitation of the inclusion of patients in this study concerns the prescription of oral chemotherapy, which is not recognized and noted in patient medical records with the same rigor as the intravenous chemotherapy. This recruitment bias shows that the traceability of oral chemotherapy and the observance of these agents are important factors for patient inclusion. This issue of oral anticancer therapies should be further investigated.

Moreover, all patients included in the study were judged fit by their oncologist for receiving intravenous chemotherapy and most patients presented with a PS of $0-1$. But at the time of the study, geriatric competence was not available routinely in the centers involved in the study. Moreover, too many data are missing to have a retrospective appraisal of the comprehensive geriatric assessment based on autonomy level, presence or lack of associated comorbidities, and cognitive or nutritional status. Although all indications were decided after multidisciplinary discussion, these limitations are inherent to a retrospective multicenter study on patients treated over almost 10 years and reflect an insufficient analysis of geriatric vulnerabilities before treatment decision.

The geriatric assessment is a multidisciplinary diagnostic evaluation that helps to precisely determine the medical, psychological, social and functional capacity of elderly patients and to elucidate subsequently their phys-

Chemotherapy in Nonagenarians iological age, as opposed to their chronological age and their capacity to receive chemotherapy $[1,10,17]$. The International Society of Geriatric Oncology and National Comprehensive Cancer Network recommend a geriatric assessment to select the best cancer treatment for an older patient with cancer [18-20].

Geriatric assessment was not reported for our patients. However, since this assessment was integrated in the pretherapeutic evaluation of elderly patients in the two institutions where our study was conducted, no patient is treated with chemotherapy if he is diagnosed beyond the age of 90 .

Through a comprehensive geriatric assessment, we can distinguish a group of elderly patients who are fit to receive standard specific anticancer treatment, and a group of vulnerable and frail patients who would benefit from modified treatment or best supportive care. Therefore, standard surgical treatment of colorectal cancer must be offered to selected elderly patients and they must receive the same complementary therapies as younger patients as well [3]. Medical optimization and excellent continuity of care may contribute to improved outcomes following surgery for these complex patients [21].

In a systemic review by Puts et al. 2012 [10], four studies, all conducted in France, examined the impact of geriatric assessment on cancer treatment [22-25]. In two studies, geriatric assessment did not influence the treatment decision, whereas in the other two studies, it led to changes in the treatment plan in $40-50 \%$ of patients.

Most of the treatment-related toxicities reported in the literature were of grade 3 or 4 and led to treatment interruption and postoperative complications [10]. Low grip strength was reported to be the only frailty marker (of seven measured) to predict treatment toxicity in a prospective observational study of 112 patients with different types of cancer [26]. Hamaker et al. [27] conducted a systematic search of studies on geriatric assessment in oncology, focusing on the association between baseline assessment and outcomes, and found that frailty was predictive of chemotherapy-induced toxicity; impairments in cognitive functions and activities of daily living were predictive of chemotherapy completion, and impairment in instrumental activities of daily living was predictive of perioperative complications. However, these results were too inconsistent to guide treatment decisions.

The geriatric assessment was able to predict mortality in multiple studies, and the variables which were found to be most associated with increased mortality are: older age, inadequate finances, mental health, comorbidities, high medication use, high Groningen Frailty Indicator 
score, low Mini-Nutritional Assessment score and impairment in activities of daily living [10,27].

A retrospective analysis [28] conducted in octogenarian and nonagenarian patients with acute myeloid lymphoma identified a near-normal albumin as favorable factor for the whole cohort, age less than 83 years and nonmonocytic morphology as favorable factors in the intensively treated cohort, and bone marrow blast $<46 \%$ as a favorable factor for patients who received supportive care.

It is recommended that treatment decisions should be discussed in a multidisciplinary setting, ideally in combination with geriatric assessment scores, comorbidity and frailty indices, scores for predicting toxicity from chemotherapy and prognostic index for survival $[4,6]$.

In fact, the nonagenarian patients who would theoretically tolerate a treatment with chemotherapy should receive comprehensive geriatric assessment in order not to compromise their autonomy, their general health conditions and their quality of life by such treatment. There is no consensus in relation to the selection and type of treatment for the group of vulnerable and the group of frail patients $[3,11]$. At present, frail elderly patients remain the real challenge and the most appropriate approach to their treatment is still unclear.

It does not seem reasonable, from ethical and economical points of view, to treat nonagenarians if the proposed treatment is not curative. However, prospective studies in vulnerable and frail patients are highly needed in order to develop guidelines between social policies and specific health care to maintain both good health and quality of life in this group of nonagenarian patients. Only a multicenter prospective study will help to provide a more accurate analysis on the clinical benefit of treating these very old and frail patients.

\section{Disclosure Statement}

There are no conflicts of interest to declare.

\section{References}

1 Pal SK, Hurria A: Impact of age, sex, and comorbidity on cancer therapy and disease progression. J Clin Oncol 2010;28:4086-4093.

2 Alon S: Psychosocial challenges of elderly patients coping with cancer. J Pediatr Hematol Oncol 2011;33:S112-S114.

3 Destri GL, Cavallaro M, Trovato MA, Ferlito F, Castaing M, Puleo S: Colorectal cancer treatment and follow-up in the elderly: an inexplicably different approach. Int Surg 2012; 97:219-223.

4 Markopoulos C, van de Water W: Older patients with breast cancer: is there bias in the treatment they receive? Ther Adv Med Oncol 2012;4:321-327.

5 Vercelli M, Lillini R, Capocaccia R, Micheli A, Coebergh JW, Quinn M, et al: Cancer survival in the elderly: effects of socio-economic factors and health care system features (ELDCARE project). Eur J Cancer 2006;42: 234-242.

6 Leo S, Accettura C, Gnoni A, Licchetta A, Giampaglia M, Mauro A, et al: Systemic treatment of gastrointestinal cancer in elderly patients. J Gastrointest Cancer 2013; 44:22-32.

7 Lagro J, Timmer-Bonte J, Maas HAAM: Predictors of early death risk in older patients treated with first-line chemotherapy for cancer and the importance of geriatric assessment. J Clin Oncol 2012;30:4443-4443.
8 Sacher AG, Le LW, Leighl NB, Coate LE: Elderly patients with advanced NSCLC in phase III clinical trials: are the elderly excluded from practice-changing trials in advanced NSCLC? J Thorac Oncol 2013;8:366-368.

9 Mohile SG, Magnuson A: Comprehensive geriatric assessment in oncology; in Extermann M (ed): Cancer and Aging: From Bench to Clinics. Interdiscipl Top Gerontol. Basel, Karger, 2013, vol 38, pp 85-103.

10 Puts MTE, Hardt J, Monette J, Girre V, Springall E, Alibhai SMH: Use of geriatric assessment for older adults in the oncology setting: a systematic review. J Natl Cancer Inst 2012; 104:1134-1164

11 Ruiz M, Reske T, Cefalu C, Estrada J: Management of elderly and frail elderly cancer patients: the importance of comprehensive geriatrics assessment and the need for guidelines. Am J Med Sci 2013;346:66-69.

12 Balducci L, Extermann M: Management of cancer in the older person: a practical approach. Oncologist 2000;5:224-237.

13 Kornblith $A B$, Kemeny M, Peterson BL, Wheeler J, Crawford J, Bartlett N, et al: Survey of oncologists' perceptions of barriers to accrual of older patients with breast carcinoma to clinical trials. Cancer 2002;95:989-996.

14 Repetto L: Greater risks of chemotherapy toxicity in elderly patients with cancer. J Support Oncol 2003;1:18-24.

15 Monfardini S: Prescribing anti-cancer drugs in elderly cancer patients. Eur J Cancer 2002; 38:2341-2346.
16 Gridelli C, Perrone F, Gallo C, Cigolari S, Rossi A, Piantedosi F, et al: Chemotherapy for elderly patients with advanced non-small-cell lung cancer: the Multicenter Italian Lung Cancer in the Elderly Study (MILES) phase III randomized trial. J Natl Cancer Inst 2003;95: 362-372.

17 Johannsen DL, DeLany JP, Frisard MI, Welsch MA, Rowley CK, Fang X, et al: Physical activity in aging: comparison among young, aged, and nonagenarian individuals. J Appl Physiol (1985) 2008;105:495-501.

18 SIOG: Comprehensive Geriatric Assessment. http://www.siog.org./index.php?option=com content\&view $=$ article\&id=245: comprehensi ve-geriatric-assessment $\&$ catid $=25$ : the project\&Itemid $=160$.

19 National Comprehensive Cancer Network. NCCN Clinical Practice Guidelines in Oncology. Senior Adult Oncology, version 2. https://www.nccn.org/store/login/login. aspx?ReturnURL=http://www.nccn.org/professionals/physician_gls/pdf/senior.pdf.

20 Droz J-P, Balducci L, Bolla M, Emberton M, Fitzpatrick JM, Joniau S, et al: Management of prostate cancer in older men: recommendations of a working group of the International Society of Geriatric Oncology. BJU Int 2010; 106:462-469.

21 Kunitake H, Zingmond DS, Ryoo J, Ko CY: Caring for octogenarian and nonagenarian patients with colorectal cancer: what should our standards and expectations be? Dis Colon Rectum 2010;53:735-743. 
22 Aparicio T, Girard L, Bouarioua N, Patry C, Legrain S, Soulé JC: A mini geriatric assessment helps treatment decision in elderly patients with digestive cancer. A pilot study. Crit Rev Oncol Hematol 2011;77:63-69.

23 Chaïbi P, Magné N, Breton S, Chebib A, Watson S, Duron J-J, et al: Influence of geriatric consultation with comprehensive geriatric assessment on final therapeutic decision in elderly cancer patients. Crit Rev Oncol Hematol 2011;79:302-307.

24 Girre V, Falcou MC, Gisselbrecht M, Gridel $\mathrm{G}$, Mosseri V, Bouleuc C, et al: Does a geriatric oncology consultation modify the cancer treatment plan for elderly patients? J Gerontol A Biol Sci Med Sci 2008;63:724-730.

25 Barthélémy P, Heitz D, Mathelin C, Polesi H, Asmane I, Litique V, et al: Adjuvant chemotherapy in elderly patients with early breast cancer. Impact of age and comprehensive geriatric assessment on tumor board proposals. Crit Rev Oncol Hematol 2011;79:196-204.

26 Puts MTE, Monette J, Girre V, Pepe C, Monette M, Assouline S, et al: Are frailty markers useful for predicting treatment toxicity and mortality in older newly diagnosed cancer patients? Results from a prospective pilot study. Crit Rev Oncol Hematol 2011;78: 138-149.

27 Hamaker ME, Vos AG, Smorenburg CH, de Rooij SE, van Munster BC: The value of geriatric assessments in predicting treatment tolerance and all-cause mortality in older patients with cancer. Oncologist 2012;17:1439-1449.

28 Harb AJ, Tan W, Wilding GE, Ford L, Sait SNJ, Block AW, et al: Treating octogenarian and nonagenarian acute myeloid leukemia patients - predictive prognostic models. Cancer 2009; 115:2472-2481. 